\section{Does child survival limit family size?}

Hans Rosling (1948-2017), physician and epidemiologist, famously upturned assumptions widely held by the public and by the development community assumptions that, thanks to US President Donald Trump, are back in the spotlight. A recurring theme of Rosling's was that family sizes have been shrinking even though child survival rates have improved (see, for example, Nature 540, 330-333; 2016). Although reducing infant mortality is an unfettered good, we are concerned that key family-planning initiatives could be lost if governments uncritically assume that it leads to people having fewer children.

Rosling's global statistics on total fertility rate and infant mortality rate do not indicate causality, neither are they necessarily correlated. Although such a correlation holds for Asia, it does not in Africa. The infant mortality rate in Niger, for example, has fallen by two-thirds since the 1980s but the country's total fertility rate has risen slightly, leading to a predicted population explosion from 20 million in 2015 to 72 million by 2050 (see go.nature.com/2j7rzcv).

In our view, President Trump's reinstatement of the Mexico City Policy (the 'global gag rule', which denies US funds to foreign non-governmental organizations associated with abortion counselling and referrals) will be disastrous for population control and for women's health. When President George W. Bush imposed a milder version of this rule in 2001, the unofficial abortion rate in African counties rose steeply (E. Bendavid et al. Bull. World Health Organ. 89, 873-880; 2011). US politicians must grasp that the best way to prevent abortions is to invest heavily in accessible family planning. Malcolm Potts, Alisha Graves University of California, Berkeley,
California, USA.

Duff Gillespie Johns Hopkins University, Maryland, USA. potts@berkeley.edu

\section{Model to accelerate epidemic responses}

On behalf of the Scientific Advisory Council of the influenza data-sharing initiative GISAID (www.gisaid.org), I suggest that the principles of this long-standing and successful programme could be extended to help speed on-the-ground responses to other emergent viral threats.

GISAID operates under a unique Database Access Agreement that governs sharing of data in its EpiFlu database without infringing intellectualproperty rights (see S. Elbe and G. Buckland-Merrett Global Challenges 1, 33-46; 2017). This enforceable agreement and the code of conduct between providers and users of data are trusted by the community, in the spirit of the World Health Organization's (WHO's) longestablished Global Influenza Surveillance and Response System and its Pandemic Influenza Preparedness Framework, which was adopted in 2011.

Open access to these latest data guides the WHO's twiceyearly recommendations on the composition of seasonal influenza vaccines. And the prompt release of H7N9 influenzavirus sequences from the first human cases in China facilitated production of candidate vaccine viruses within a few weeks

(P. R. Dormitzer et al. Sci. Transl. Med. 5, 185ra68; 2013).

The latest advances in rapid gene sequencing mean that the GISAID model could now be adapted for other dangerous viruses, such as those that cause Middle East respiratory syndrome, Ebola haemorrhagic fever and Zika virus disease (see N. L. Yozwiak et al. Nature 518, 477-479; 2015).
John W. McCauley The Francis Crick Institute, London, UK. john.mccauley@crick.ac.uk

\section{Locals embrace China nuclear project}

Public support for China's nuclearenergy programme plummeted after Japan's Fukushima disaster in 2011. So far, however, there have been no reported protests against a proposed nuclear power plant in Zhangzhou in southeast China. Other nations could learn from the strategy used by the authorities to make this project acceptable to the public.

After announcing the proposal in October last year, China's National Nuclear Corporation and the regional government engaged with local communities to communicate the planning issues and improve transparency. They also offered support for potential job seekers and for local enterprises. These efforts were spurred by the suspension two months earlier of a proposed nuclear plant in Lianyungang, Jiangsu Province, in response to public outcry.

Support for the Zhangzhou project has been boosted by the prospect of better infrastructure and more employment, and because no resettlements are necessary. Residents were invited to visit the Daya Bay nuclear plant to allay their fears. Although Zhangzhou is in a coastal seismic fault zone, engineering can help to safeguard the plant against earthquakes. The risk of tsunami damage is reduced because the site will be 13.5 metres above sea level.

In our view, the project should nevertheless be subject to morestringent construction permits and stricter safety measures than nuclear plants on sites that are less potentially hazardous. Hong Yang Norwegian Institute of Bioeconomy Research, Norway. Junqiang Xia Wuhan University, China.

Roger J. Flower University

College London, UK.

hongyanghy@gmail.com

\section{Award bonus points to motivate reviewers}

The proliferation of scientific journals is making it harder for editors to recruit peer reviewers. Various incentives (see, for example, Nature 514, 274; 2014) have not substantially changed an archaic system. I propose a smart solution that would benefit reviewers, authors and journals - namely, to award bonus points to reviewers for use towards their own open-access publishing fees.

An estimated 63 million hours were devoted to peer review in 2015 (M. Kovanis et al. PLoS ONE 11, e0166387; 2016). However, most journals still continue to thank reviewers merely through an automatic e-mail. Under my proposed system, journals would award points as e-coupons to reviewers according to the quality of their review and the effort they invested in it.

Individual points would accumulate and be tracked to avoid mismatches between reviewers with similar names. Co-authors could combine their own reviewer points to contribute towards publication costs. Reciprocal agreements would allow transfer of points between publishers, but not between individuals.

This reward system would encourage reviewers, potentially enlarging the referee pool. It would also improve review quality and speed up the review process. Because the scheme promotes open-access publication, publishers' revenues would be minimally affected or even increased.

David Gurwitz Tel-Aviv

University, Israel. gurwitz@post.tau.ac.il

CONTRIBUTIONS

Correspondence may be sent to correspondence@ nature.com after consulting the author guidelines at http://go.nature.com/ cmchno. 